\title{
Drug dosage recommendations in patients with chronic liver disease
}

\author{
Leonor Periáñez-Párraga, Iciar Martínez-López, Pere Ventayol-Bosch, Francesc Puigventós-Latorre \\ and Olga Delgado-Sánchez
}

Department of Pharmacy. Hospital Universitari Son Espases. Palma de Mallorca-Illes Balears, Spain

\begin{abstract}
Chronic liver diseases (CLD) alter the kinetics of drugs. Despite dosage adjustment is based on Child-Pugh scores, there are no available recommendations and/or algorithms of reference to facilitate dosage regimens.

A literature review about dose adjustment of the drugs from the hospital guide -which are included in the list of the WHO recommended drugs to be avoided or used with caution in patients with liver disease- was carried out. The therapeutic novelties from the last few years were also included. In order to do so, the summary of product characteristics (SPC), the database DrugDexMicromedex, the WHO recommendations and the review articles from the last 10 years in Medline were reviewed. Moreover, the kinetic parameters of each drug were calculated with the aim of establishing a theoretical recommendation based on the proposal of Delcò and Huet.

Recommendations for 186 drugs are presented according to the SPC (49.5\%), DrugDex-Micromedex (26.3\%) and WHO (18.8\%) indications; six recommendations were based on specific publications; the theoretical recommendation based on pharmacokinetic parameters was proposed in four drugs.

The final recommendations for clinical management were: dosage modification (26.9\%), hepatic/analytical monitoring of the patient (8.6\%), contraindication (18.8\%), use with caution (19.3\%) and no adjustment required (26.3\%).

In this review, specific recommendations for the practical management of patients with chronic liver disease are presented. It has been elaborated through a synthesis of the published bibliography and completed by following a theoretical methodology.
\end{abstract}

Key words: Liver disease. Liver dysfunction. Prescription drugs. Hepatic impairment. Dose-response relationship.

Declaration of funding sources: this work has been funded by the Joaquim Bonal 2009 grant from the "Societat Catalana de Farmacia Clínica".

Received: 28-09-11.

Accepted: 23-11-11.

Correspondence: Leonor Periáñez-Párraga. Department of Pharmacy. Hospital Universitari Son Espases. Carretera Valldemosa, 79. 07010 Palma de Mallorca, Spain.

e-mail: leonord.perianez@ssib.es
Periáñez-Párraga L, Martínez-López I, Ventayol-Bosch P, Puiguentós Latorre F, Delgado-Sánchez O. Drug dosage recommendations in patients with chronic liver disease. Rev Esp Enferm Dig 2012; 104: $165-184$.

\section{INTRODUCTION}

The liver plays a fundamental role in the metabolism of most drugs because hepatic and biliary excretion processes determine the rate of their elimination from the body, while bioavailability is affected by first-pass mechanisms.

Alterations affecting the kinetics of drugs in chronic liver disease (CLD) are mainly due to three mechanisms: reduction of portal blood flow which affects the pre-systemic elimination of high extraction drugs; decreased synthesis of transport proteins, mainly albumin and alpha-glycoprotein, which affect the bioavailability of drugs highly bound to plasma proteins; reduced drug-metabolizing hepatic enzymes, which affects the amount of plasma active metabolite, thus, the effectiveness and toxicity (1).

Chronic liver disease is assessed through Child-Pugh score system which is based on five variables: the presence of ascites and encephalopathy, plasma concentrations of bilirubin and albumin and prothrombin time. The ChildPugh score indicates the level of chronic hepatic damage: score 5-6 is class A (mild); 7-9 corresponds to class B (moderate); and 10-15 is class C (severe) $(2,3)$.

Another classification scheme such as MELD (Model for End stage Liver Disease) is based on serum bilirubin concentration, serum creatinine, the international normalized ratio (INR) of prothrombin time, and the underlying cause of liver disease (4). The MELD score was designed to predict 3-month mortality among patients on a livertransplant waiting list and has been adopted to use for allocating priorities in patients awaiting liver transplantation (5). However, unlike in renal patients, where estimates of glomerular filtration rate (creatinine clearance, 
inulin clearance) correlate with kinetic parameters of drug elimination such as renal clearance, these classification schemes lack the sensitivity to quantitate the specific ability of the liver to metabolize individual drugs. That is why it is not a frequently used classification scheme for pharmacological adjustment.

The recommendations for drug dosage adjustment in patients with CLD are based on Child-Pugh scores. Since 2003 and 2005 respectively, the regulating agencies Food and Drug Administration (FDA) and the European Medicines Agency (EMA) require the performance of kinetic studies previous to the authorization of new drugs in patients with CLD in order to provide future dose adjustment recommendations $(6,7)$. This information reflected in the summary of product characteristics (SPC) is highly useful. However, a great amount of drugs lack of this specific information because patients recruited in clinical trials show a good hepatic function (Child-Pug class A) and do not have an advanced liver dysfunction (Child-Pugh class $\mathrm{C}$ ).

The complexity of hepatic metabolism has limited the development of tools allowing predictions of drug behavior in patients with chronic liver disease. Unlike in renal insufficiency, there are no guidelines and algorithms of reference to facilitate drug dosage in these patients. In this context, there is a growing need to check and expand the available information in the SPC. The review of recommendations from other sources and the application of calculation procedures based on the kinetic parameters of the drug are needed to establish practical dose recommendations in CLD for the frequently used drugs in the care environment.

The aim of this article is to provide dose adjustment recommendations for the most commonly used drugs in the hospital in patients with chronic liver disease.

\section{MATERIALS AND METHODS}

Drugs were selected from the list of medicines WHO recommends to avoid or to use with caution in patients with liver disease (8), selecting those included in the hospital Pharmacotherapeutic Guide (PG). The therapeutic novelties from over the last few years were also included.

A literature review about dosage recommendations in chronic liver disease was carried out for the selected drugs from the following sources: a) SPC; b) DrugDex Micromedex (9); c) WHO recommendations (8); and d) review articles published in the last 10 years in the Medline database with the following search strategy: "Hepatic"[Ti] OR "liver"[Ti]) AND ("Dose-Response Relationship, Drug"[Mesh]) AND (Review[ptyp] AND (English[lang] OR Spanish[lang]) AND “2001/02/27”[PDat]: “2011/02/24”[PDat]). The following search engines were used: "Scholar-google" and "Alquimia" (10) with the aim of finding bulletins published by centers of reference which include drug dosage recommendations in chronic liver disease.
Table I. Categorization and dose recommendation in IH patients. Categorization based on Huet et al. (11) and Krähenbühl et al. (12). Recommendation for initial dose adjustment and maintenance adapted from Delcò et al. (1)

\begin{tabular}{|c|c|c|c|c|}
\hline Category & $E_{H}$ & $F$ & $P B$ & General recommendation \\
\hline 1 & $\operatorname{High}(\geq 60 \%)$ & $\leq 40 \%$ & Any & $\begin{array}{l}\text { Reduction Id and Md by: dose } \\
\text { reduction }=(\mathrm{Nd} \times \mathrm{F}) / 100\end{array}$ \\
\hline 2 & $\begin{array}{l}\text { Intermediate } \\
(30-60 \%)\end{array}$ & $40-70 \%$ & Any & $\begin{array}{l}\text { Id: start in the low range } \\
\text { of normal } \\
\text { Md: should be adjusted as } \\
\text { described in low } E_{H} \text { and } \\
\text { low PB }\end{array}$ \\
\hline 3 & Low $(\leq 30 \%)$ & $\geq 70 \%$ & $\begin{array}{l}\geq 90 \% \\
<90 \%\end{array}$ & $\begin{array}{l}\text { Drug monitoring } \\
\text { Md: } \\
\text { CP A: } 50 \% \text { of } \mathrm{Nd} \\
\text { CP B: } 25 \% \text { of } \mathrm{Nd} \\
\text { CP C: drug monitoring }\end{array}$ \\
\hline
\end{tabular}

$4 \quad$ Unknown

Id: initial dose. Md: maintenance dose. Nd: normal dose without liver disease. EH hepatic extraction ratio. F: bioavailability. BP: fraction bounds to proteins. CP: ChildPugh index.

The final dosage recommendations have been based on the SPC information and on the rest of the bibliography defined in the search strategy. In case they were not coincident, the most restrictive proposal was taken.

When no information was found, the adjustment recommendations were given according to the process defined by Delcò et al. (1), based on the method established by Huet et al. (11) and Krähenbühl et al. (12). This method classifies each drug according to three parameters: hepatic extraction ratio $\left(\mathrm{E}_{\mathrm{H}}\right)$-classified in 3 categories (high $\geq 60 \%$, intermediate $30-60 \%$ and low $\leq 30 \%$ ), bioavailability (F) and plasma protein binding (PB) (Table I). For the drugs with no $E_{H}$ available information, $E_{H}$ was calculated using the formula defined by Westphal et al. (11): $\left[\mathrm{E}_{\mathrm{H}}=\left(\mathrm{Q}_{0} \times \mathrm{CL}_{\mathrm{sys}}\right) / \mathrm{Q}_{\mathrm{H}}\right] \cdot \mathrm{Q}_{0}$ values (extra renal drug moiety) and $\mathrm{CL}_{\text {syst }}$ (systemic or total clearance) were obtained from the literature, assuming a hepatic blood flow $\left(\mathrm{Q}_{\mathrm{H}}\right)$ of $1.5 \mathrm{~L} / \mathrm{min}$.

In order to have supplementary information to the bibliography, the following parameters were registered for all drugs: $\mathrm{E}_{\mathrm{H}}$, bioavailability, plasma protein binding and the corresponding category according to the Huet classification (11).

\section{RESULTS}

The information of 191 drugs was reviewed: 56 of oncology therapy, 84 anti-infective agents [antibiotics 
(35), anti-tuberculosis drugs (5), antifungal (15) and antiretroviral agents (29)], 13 of cardiovascular therapy and 38 of other pharmacological groups.

The final recommendation was established for 186 reviewed drugs and was defined according to the SPC in 92 (49.5\%), 49 in DrugDex Micromedex (26.3\%) and 35 in WHO (18.8\%); the recommendations of 6 drugs (3.2\%) were based on the publications of Azanza et al. (14), for the group of antimicrobials. In four drugs $(2.2 \%)$ (dacarbazine, leuprorelin, maraviroc and zidovudine) no information in the previously mentioned databases was found nor in further bibliography searches. That is why a theoretical adjustment recommendation based on the proposal defined by Delcò et al. (1) and Huet et al. (11) was given. In five drugs (buserelin, dactinomycin, enfuvirtide, megestrol and trastuzumab) no information was found about dosage adjustment due to lack of data.

Depending on the type of recommendation the following was obtained: 48 drugs (25.8\%) needed quantitative adjustment; 88 drugs (47.3\%) had to follow qualitative recommendations divided into: 37 caution of use, 16 monitoring the patient and 35 contraindication; and 50 drugs $(26.9 \%)$ did not require dosage adjustment, being $72 \%$ of them of low $\mathrm{E}_{\mathrm{H}}(<0.3)$.

Regarding the reviewed drugs, the established classification by Huet et al. (11) was the following respectively: $19(10.2 \%)$ class $1 ; 28(15.1 \%)$ class $2 ; 77(41.4 \%)$ class 3 and $62(33.3 \%)$ class 4.

From the 191 reviewed drugs, $\mathrm{E}_{\mathrm{H}}$ was calculated with the Westphal et al. formula (13) in 103 drugs (53.9\%); in 66 drugs (34.6\%) it was not obtained due to lack of data; in 22 drugs $(11.5 \%) \mathrm{E}_{\mathrm{H}}$ was obtained through the bibliography.

Some other registered parameters to justify dosage adjustment were: $\mathrm{PB} \geq 90 \%$ in 66 drugs $(34.6 \%),<90 \%$ in 102 drugs $(53.4 \%)$ and no data was obtained in 23 drugs $(12.0 \%)$. The hepatic/biliary metabolism predominance with a score of $\mathrm{Q}_{0}>0.4$ was found in 65 drugs $(34.0 \%)$, it was less than 0.4 in 35 drugs $(18.3 \%)$ and it could not be obtained in $91(47.6 \%)$, which indicates that most of the drugs in which $\mathrm{Q}_{0}$ was obtained are highly metabolized and/or are excreted through bile.

The dosage adjustment recommendation is shown in table II, it indicates the final recommendation in drugs requiring adjustment in chronic liver disease, drugs in which hepatic or analytic monitorization is recommended, contraindicated drugs in chronic liver disease, drugs used with caution and drugs not requiring adjustment in chronic liver disease. The category Huet and Krähenbühl and the $\mathrm{E}_{\mathrm{H}}$ of every drug is also found in table II.

\section{DISCUSSION}

In this review, a dosage adjustment for 186 frequently used drugs in hospitals in patients with chronic liver disease is proposed.
There is not an established method available to assess hepatic failure which correlates with the hepatic clearance of drugs. Besides, the semiquantitive scale of Child-Pugh is not precise and it does not always quantify the specific capacity of the liver to metabolize different drugs. Drug adjustment to the hepatic function of the patient by using the Child-Pugh score has limitations because it was initially designed to stratify the risk of anastomosis or portocaval shunt in cirrhotic patients. However, it has been demonstrated that the Child-Pugh score shows also some relation with survival and with the development of complications in cirrhosis. The Child-Pugh score requires quantification of subjective variables such as ascites and encephalopathy, which vary among different observers and can be modified by medical interventions such as the lactulose and/or diuretics prescription in encephalopathy and ascites.

The Child-Pugh score is mostly used in the review articles which include drug dosage recommendations in patients with chronic liver disease through the application of an explicit methodological procedure $(8,15-19)$. The bulletin written by Shapiro (20) compared the Child-Pugh scores with the Cockcroft-Gault equation used in the adjustment for renal insufficiency. In some studies, the adjustment based on the $\mathrm{E}_{\mathrm{H}}$ with supplementary information such as plasma protein binding, hepatic cytochrome metabolism or transaminases among others $(1,17,19)$ is proposed. However, general recommendations are proposed for most of the drugs.

To define the drugs that have to be adjusted in patients with chronic liver disease, the main parameter proposed by different authors is $\mathrm{E}_{\mathrm{H}}$. Kim et al. (21) proposes to establish three categories to adjust drugs depending on $E_{H}$ in the hepatic first-pass effect, being those of high extraction the adjusted ones.

The recommendations of the Drug Information Service (22) propose that in the case of a severe liver dysfunction (albumin $<30 \mathrm{~g} / \mathrm{dL}, \mathrm{INR}>1.2$ ), drugs with a high $\mathrm{E}_{\mathrm{H}}$ are still the ones which should have a higher adjustment despite it includes the possibility of dose reduction in the low extraction ones. Accordingly, Shlatter et al. (16) agree in the dosage recommendations based on $\mathrm{E}_{\mathrm{H}}$ and/or pharmacological bioavailability, justifying adjustments of initial doses and maintenance.

The established recommendations about the possible dose adjustment in patients with hepatic insufficiency by Verbeeck et al. (19) are very similar to the ones by Delcò et al. (1) and Klotz (23). Both characterize the drugs depending on the hepatic extraction rate, plasma protein binding and their hydrophilicity. The difference with the previous authors is that the latest propose an initial and maintenance dose adjustment $(1,19)$ of the drugs with a low level of extraction and a narrow therapeutic range. Pirmohamed (17) proposes to select those with a wide therapeutic range to ensure a lower hepatotoxicity. Some other authors such as Sloss et al. (18) establish the avoidance of use or the increase of administration intervals in 
Table II. Recommendations for drug dosage in patients with chronic liver disease

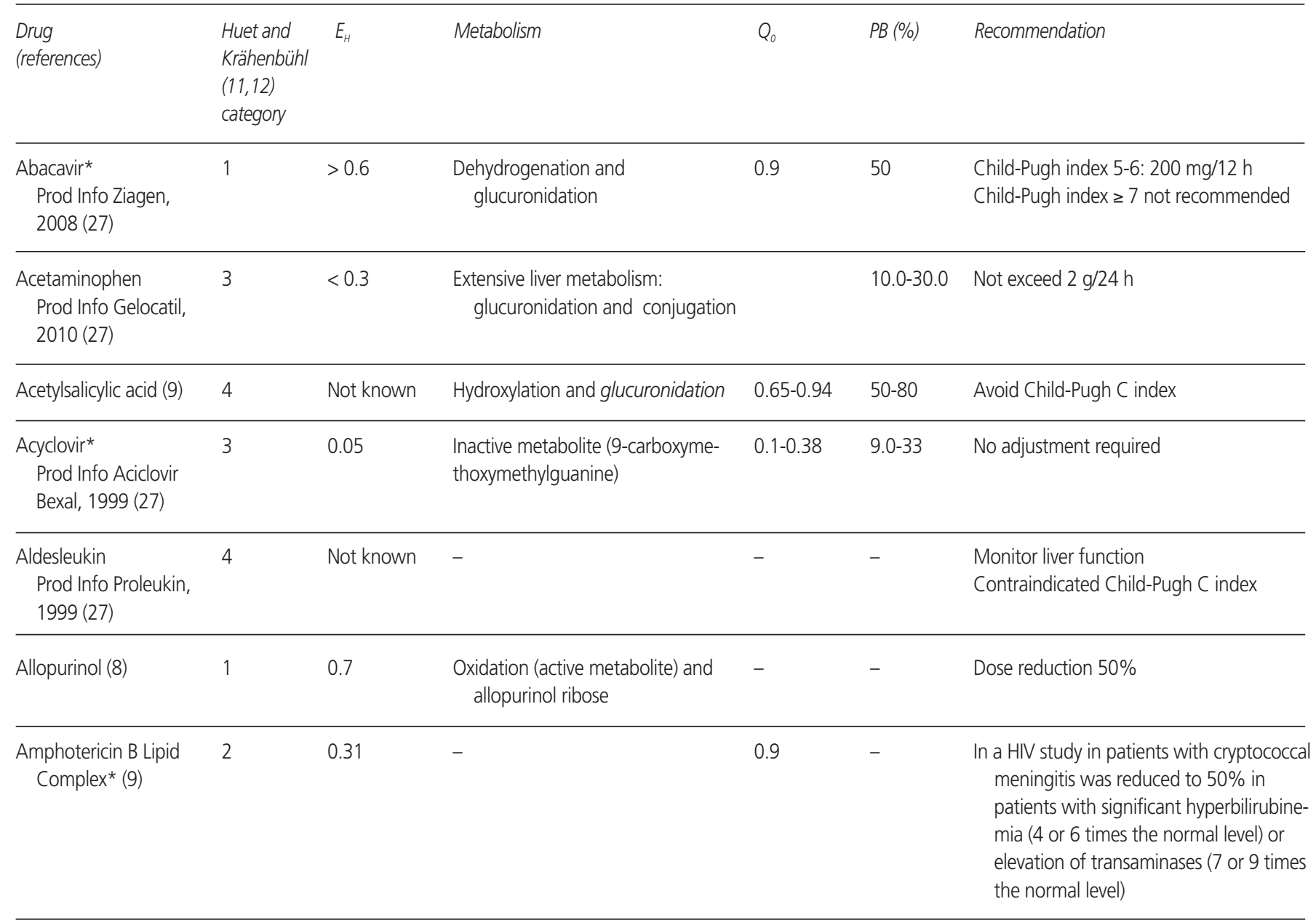

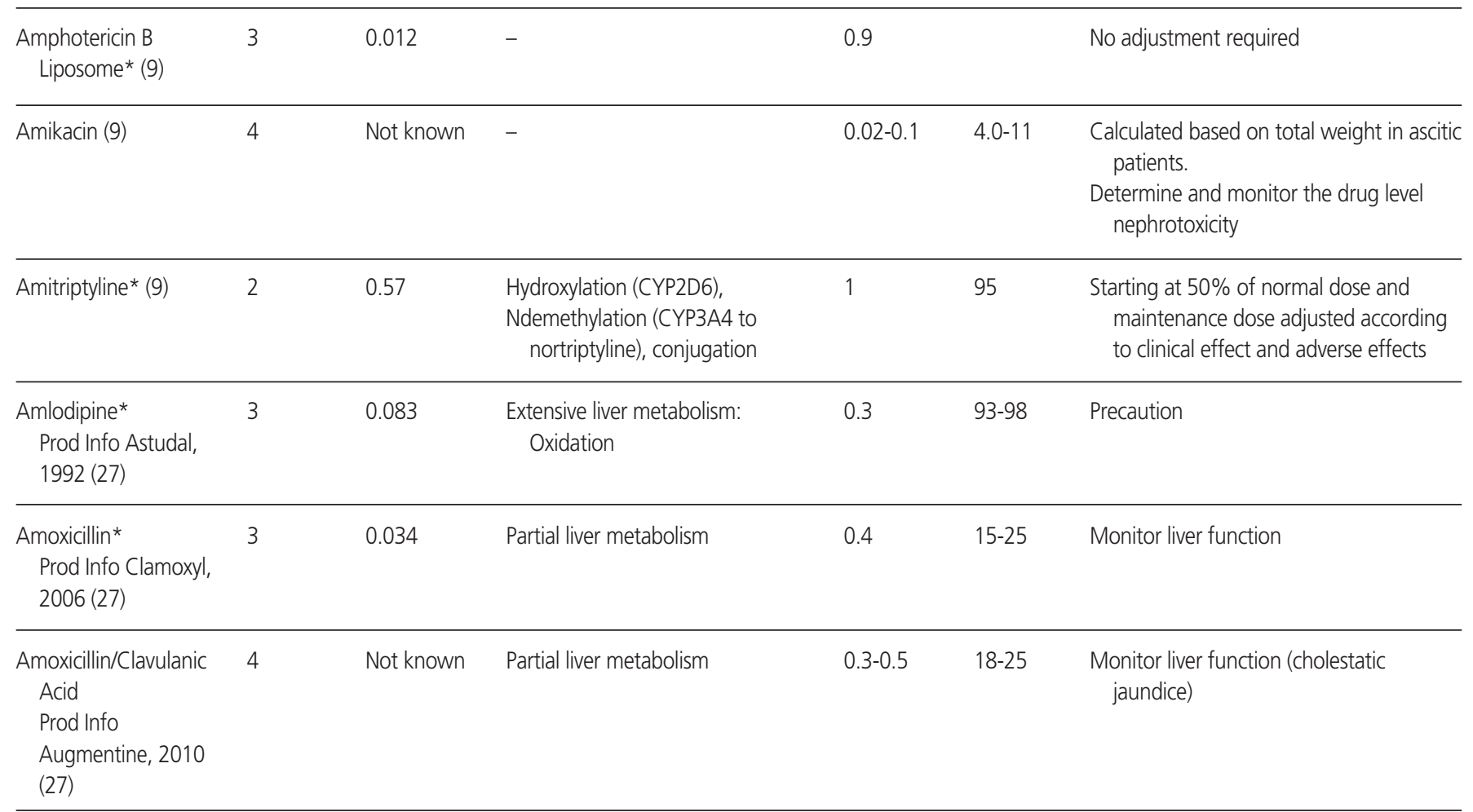


Table II (Cont.). Recommendations for drug dosage in patients with chronic liver disease

\begin{tabular}{|c|c|c|c|c|c|c|}
\hline $\begin{array}{l}\text { Drug } \\
\text { (references) }\end{array}$ & $\begin{array}{l}\text { Huet and } \\
\text { Krähenbühl } \\
(11,12) \\
\text { category }\end{array}$ & $E_{H}$ & Metabolism & $Q_{0}$ & $P B(\%)$ & Recommendation \\
\hline $\begin{array}{l}\text { Ampicillin* } \\
\text { Prod Info Ampilevel, } \\
1999(27)\end{array}$ & 3 & 0.037 & - & 0.2 & $17-20$ & Precaution \\
\hline Ampicillin/sulbactam (9) & 4 & Not known & - & 0.3 & 17 & Precaution \\
\hline $\begin{array}{l}\text { Anastrazole } \\
\text { Prod Info Arimidex, } \\
2010(27)\end{array}$ & 4 & Not known & $\begin{array}{l}\text { N-dealkylation, hydroxylation (CYP), } \\
\text { glucuronidation }\end{array}$ & 0.95 & 45 & No adjustment required \\
\hline $\begin{array}{l}\text { Anidulafungin* } \\
\text { Prod Info Ecalta, } 2007 \\
(27)\end{array}$ & 3 & 0.003 & Not undergo hepatic metabolism & 0.3 & 99 & No adjustment required \\
\hline $\begin{array}{l}\text { Artemether/ } \\
\text { Lumefantrine (8) }\end{array}$ & 4 & Not known & $\begin{array}{l}\text { Artemether: CYP450 (CYP3A4/5, } \\
\text { CYP2B6, CYP2C9, CYP2C19). } \\
\text { Dihydroartemisinin (DHA), an } \\
\text { active metabolite } \\
\text { Lumefantrine: CYP450 (CYP3A4, } \\
\text { CYP2D6) }\end{array}$ & & $95-99$ & Precaution in Child Pugh C index \\
\hline
\end{tabular}

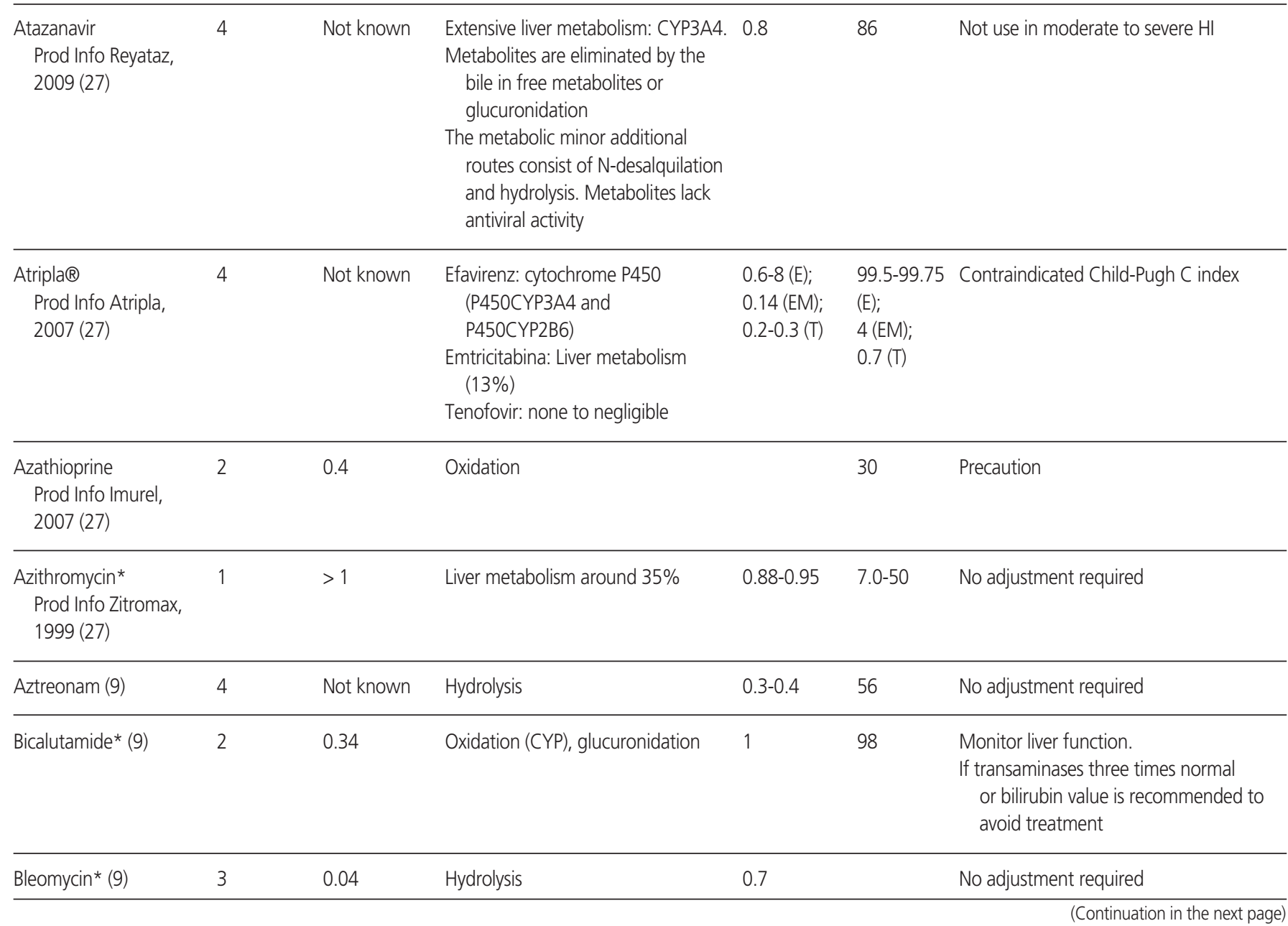


Table II (Cont.). Recommendations for drug dosage in patients with chronic liver disease

\begin{tabular}{|c|c|c|c|c|c|c|}
\hline $\begin{array}{l}\text { Drug } \\
\text { (references) }\end{array}$ & $\begin{array}{l}\text { Huet and } \\
\text { Krähenbühl } \\
(11,12) \\
\text { category }\end{array}$ & $E_{H}$ & Metabolism & $Q_{0}$ & $P B(\%)$ & Recommendation \\
\hline Bupivacaine* (8) & 2 & 0.31 & Glucuronidation & 0.9 & 95 & Avoid Child-Pugh C index \\
\hline Buserelin & 3 & Not known & - & & & Not calculable \\
\hline $\begin{array}{l}\text { Busulfan* } \\
\text { Prod Info Busilvex, } \\
2008 \text { (27) }\end{array}$ & 3 & 0.21 & Oxidation, sulfation & 1 & 30 & Precaution \\
\hline $\begin{array}{l}\text { Capecitabine* }^{*} \\
\text { Prod Info Xeloda, } \\
2001(27)\end{array}$ & 1 & 2.7 & $\begin{array}{l}\text { Carboxylesterase, cytidine } \\
\text { desaminase, phosphorylation }\end{array}$ & 0.97 & 54 & No adjustment required \\
\hline $\begin{array}{l}\text { Carboplatin* } \\
\text { Prod Info Carboplati- } \\
\text { no Teva, } 2009 \text { (27) }\end{array}$ & 3 & 0.012 & - & 0.25 & 20 & $\begin{array}{l}\text { No adjustment required. If overdose } \\
\text { occurs hepatotoxicity }\end{array}$ \\
\hline $\begin{array}{l}\text { Caspofungin* } \\
\text { Prod Info Cancidas, } \\
2009(27)\end{array}$ & 3 & 0.004 & $\mathrm{~N}$-acetylation and hydrolysis & 0.59 & 97 & $\begin{array}{l}\text { Child-Pugh index = 7-9: } 70 \mathrm{mg} \\
\text { administered the first day, then } \\
35 \mathrm{mg} / 24 \text { recommends continuing } \\
\text { Child-Pugh index } \geq 10 \text { : initial } \\
\text { and maintenance dose } \\
35 \mathrm{mg} / 24 \mathrm{~h}\end{array}$ \\
\hline
\end{tabular}

\begin{tabular}{|c|c|c|c|c|c|c|}
\hline $\begin{array}{l}\text { Cefazolin* } \\
\text { Prod Info Cefazolina } \\
\text { normon, } 2002 \text { (27) }\end{array}$ & 3 & 0.008 & Minimal liver metabolism & $0-0.4$ & $80-86$ & No adjustment required \\
\hline
\end{tabular}

Cefditoren
$\begin{aligned} & \text { Prod Info Spectracef, } \\ & 2010(27)\end{aligned}$

\begin{tabular}{|c|c|c|c|c|c|c|}
\hline $\begin{array}{l}\text { Cefepime* } \\
\text { Prod Info Maxipime, } \\
1999(27)\end{array}$ & 3 & 0.012 & $\begin{array}{l}\text { Partial liver metabolism. } 85 \% \text { is } \\
\text { excreted as unchanged }\end{array}$ & $0.01-0.3$ & $16-20$ & No adjustment required \\
\hline
\end{tabular}

\begin{tabular}{lllllll}
\hline Cefotaxime (9) & 4 & Not known & Desacetyl & $0.15-0.5$ & $27-38$ & No adjustment required \\
\hline $\begin{array}{l}\text { Cefoxitin* (9) } \\
\text { (9) }\end{array}$ & 0.025 & $\begin{array}{c}\text { Minimal liver metabolism (2\%): } \\
\text { descarbamyl, inactive } \\
\text { metabolite }\end{array}$ & $0.1-0.15$ & $41-75$ & No adjustment required \\
\hline $\begin{array}{l}\text { Ceftazidime* } \\
\text { Prod Info Fortaz, }\end{array}$ & 3 & 0.007 & Not undergo hepatic metabolism & $0.04-0.1$ & $5.0-17$ & No adjustment required \\
\hline $\begin{array}{l}\text { Ceftriaxone* (9) } \\
\text { (27) }\end{array}$ & 3 & 0.04 & Minimal liver metabolism & $0.33-0.67$ & $83-96$ & No adjustment required \\
\hline
\end{tabular}


Table II (Cont.). Recommendations for drug dosage in patients with chronic liver disease

\begin{tabular}{|c|c|c|c|c|c|c|}
\hline $\begin{array}{l}\text { Drug } \\
\text { (references) }\end{array}$ & $\begin{array}{l}\text { Huet and } \\
\text { Krähenbühl } \\
(11,12) \\
\text { category }\end{array}$ & $E_{H}$ & Metabolism & $Q_{0}$ & $P B(\%)$ & Recommendation \\
\hline $\begin{array}{l}\text { Cefuroxime* }^{*} \\
\text { Prod Info Zinnat, } \\
2008(27)\end{array}$ & 3 & 0.048 & Nonspecific esterases & $0-0.34$ & 50 & No adjustment required \\
\hline Chlorambucil (8) & 3 & $<0.3$ & Extensive liver metabolism & 1 & 99 & Precaution \\
\hline $\begin{array}{l}\text { Ciprofloxacin } \\
\text { Prod Info Baycip, } \\
2010(27)\end{array}$ & 2 & 0.4 & $\begin{array}{l}\text { Hepatic metabolism results in } 4 \\
\text { metabolites have been identified } \\
\text { as desetilenciprofloxacin, } \\
\text { sulfociprofloxacin, and } \\
\text { formylciprofloxacin oxociprofloxa- } \\
\text { cin. The first three have an } \\
\text { antibacterial activity comparable } \\
\text { to or lower than nalidixic acid. The } \\
\text { last is largely equivalent to } \\
\text { norfloxacin in their antimicrobial } \\
\text { activity }\end{array}$ & $0.43-0.7$ & $20-40$ & No adjustment required \\
\hline Cisplatin* (9) & 3 & 0.002 & - & 0.65 & 90 & No adjustment required \\
\hline $\begin{array}{l}\text { Cladribine } \\
\text { Prod Info Litak, } 2009 \\
\text { (27) }\end{array}$ & 4 & Not known & - & & 25 & Contraindicated \\
\hline Clarithromycin* (9) & 3 & 0.29 & Hydroxylation & $0.6-0.8$ & $>90$ & No adjustment required \\
\hline Clindamycin (8) & 3 & $<0.3$ & $\begin{array}{l}\text { Extensive liver metabolism: Actives } \\
\text { metabolites (N-dimethyland } \\
\text { sulfoxide) }\end{array}$ & $0.32-0.95$ & $60-95$ & $\begin{array}{l}\text { Monitor liver function. } \\
\text { In Child-Pugh C increase interval doses or } \\
\text { decrease dosing }\end{array}$ \\
\hline Clomipramine* (8) & 2 & 0.5 & $\begin{array}{l}\text { Hydroxylation } \\
\text { by CYP2D6, demethylation } \\
\text { to active metabolite } \\
\text { (N-Desmethylclomipramine) by } \\
\text { CYP3A4, CYP2C19 and CYP 1A2, } \\
\text { glucuronidation }\end{array}$ & 1 & 98 & Avoid Child-Pugh C index \\
\hline
\end{tabular}

\begin{tabular}{|c|c|c|c|c|c|c|}
\hline Cloxacillin* (8) & 3 & 0.035 & Liver metabolism around $12 \%$ & $0.3-0.6$ & $94-95$ & Precaution \\
\hline Codeine (8) & 2 & 0.52 & $\begin{array}{l}\text { Extensive liver metabolism } \\
\quad(24-89 \%) . \\
\text { Glucuronidation }\end{array}$ & 0.1 & 0 & $\begin{array}{l}\text { Avoid Child-Pugh C index and cirrhosis, } \\
\text { produces sedation }\end{array}$ \\
\hline $\begin{array}{l}\text { Combivir® } \\
\text { Prod Info Combivir, } \\
2009(27)\end{array}$ & 4 & Not known & $\begin{array}{l}\text { Zidovudine: metabolized in the liver } \\
\quad(60 \%) \text {, forming an inactive } \\
\text { glucuronide conjugate antiviral } \\
\text { Lamivudine: minimal liver } \\
\text { metabolism }(5-10 \%)\end{array}$ & $\begin{array}{l}0.1(\mathrm{Z}) ; \\
0.3(\mathrm{~L})\end{array}$ & $<36-38$ & $\begin{array}{l}\text { Recommend drugs separately. Avoid if } \\
\text { acute dysfunction }\end{array}$ \\
\hline Cyclophosphamide* (8) & 3 & 0.04 & $\begin{array}{l}\text { Hydroxylation (CYPs 2B6, 2C19, } \\
\text { 2C9, 3A4) }\end{array}$ & 0.9 & 15 & $\begin{array}{l}\text { If total bilirubin } \geq 3 \mathrm{mg} / \mathrm{ml} \text { reduce dose by } \\
25 \% \\
\text { Monitor liver function }\end{array}$ \\
\hline
\end{tabular}


Table II (Cont.). Recommendations for drug dosage in patients with chronic liver disease

\begin{tabular}{|c|c|c|c|c|c|c|}
\hline $\begin{array}{l}\text { Drug } \\
\text { (references) }\end{array}$ & $\begin{array}{l}\text { Huet and } \\
\text { Krähenbühl } \\
(11,12) \\
\text { category }\end{array}$ & $E_{H}$ & Metabolism & $Q_{0}$ & $P B(\%)$ & Recommendation \\
\hline $\begin{array}{l}\text { Cyclosporin } \\
\text { Prod Info Sandimmun } \\
\text { neoral, } 2010(27)\end{array}$ & 1 & 0.72 & Cytochrome P-450: CYP3Al & 0.9 & 90 & Precaution. Determine drug level \\
\hline Cytarabine* $(9)$ & 2 & 0.55 & Cytidine deaminase & 0.9 & 13 & $\begin{array}{l}\text { If total bilirubin }>2 \mathrm{mg} / \mathrm{ml} \text { reduce dose } \\
\text { by } 50 \% \\
\text { Monitor liver function }\end{array}$ \\
\hline Dacarbazine* & 3 & 0.04 & $\begin{array}{l}\text { Extensive liver metabolism causing } \\
\text { some metabolites with cytotoxic } \\
\text { activity, being eliminated in the } \\
\text { urine } 18-63 \%\end{array}$ & 0.3 & 5 & $\begin{array}{l}\text { ** Maintenance dose: } \\
\text { CP A: } 50 \% \text { of normal dose } \\
\text { CP B: } 25 \% \text { of normal dose } \\
\text { CP C: drug monitoring }\end{array}$ \\
\hline
\end{tabular}

\begin{tabular}{|c|c|c|c|c|c|c|}
\hline Dactinomycin & 4 & Not known & Biliar excretion: $50-90 \%$ & 0.7 & & Not calculable \\
\hline $\begin{array}{l}\text { Daptomycin* } \\
\text { Prod Info Cubicin, } \\
2006(27)\end{array}$ & 3 & 0.002 & $\begin{array}{l}\text { Studies in vitro found that } \\
\text { daptomycin was not metabolized } \\
\text { by human liver microsomes }\end{array}$ & 0.2 & $90-95$ & Precaution in Child-Pugh C index \\
\hline $\begin{array}{l}\text { Darunavir } \\
\text { Prod Info Prezista, } \\
2010(27)\end{array}$ & 4 & Not known & Oxidation (CYP3A4) & 0.79 & 95 & $\begin{array}{l}\text { Child-Pugh index }<10 \text { No adjustment } \\
\text { required } \\
\text { Child-Pugh index }>10 \text { not } \\
\text { recommended }\end{array}$ \\
\hline
\end{tabular}

\begin{tabular}{lll}
\hline Daunorubicin (9) $\quad 4 \quad$ Not known & $\begin{array}{l}\text { Mainly liver: formation of a metaboli } 0.9 \\
\text { te with cytotoxic activity } \\
\text { (daunorubicinol), glucuronide, } \\
\text { sulfate and aglycones }\end{array}$ & $\begin{array}{l}\text { If total bilirubin }>1.5-3 \text { reduce } 25 \% \\
\text { If total bilirubin }>3 \text { reduce } 50 \%\end{array}$ \\
& &
\end{tabular}

\begin{tabular}{|c|c|c|c|c|c|c|}
\hline Diazepam* (9) & 3 & 0.02 & $\begin{array}{l}\text { Extensive liver metabolism: } \\
\text { N-demethylation (CYP2C 19), } \\
\text { hydroxylation (CYP 3A4), } \\
\text { glucuronidation } \\
\text { Ndesmethyldiazepam, oxazepam } \\
\text { and temazepam are active } \\
\text { metabolites }\end{array}$ & 1 & 98 & Reduce dose to $50 \%$ or use lorazepam \\
\hline Docetaxel* $^{*}(9)$ & 2 & 0.43 & $\begin{array}{l}\text { Oxidation (CYP3A4). } \\
\text { Biliar excretion: } 75 \%\end{array}$ & 1 & 95 & $\begin{array}{l}\text { If transaminase }>1.5 \text { normal value or } \\
\text { alkaline phosphatase }>2.5 \text { normal } \\
\text { value to reduce the dose } \\
\text { by } 25 \% \\
\text { Do not administered if serum bilirubin } \\
\text { increased or transaminase }>3.5 \\
\text { normal value or alkaline } \\
\text { phosphatase }>6 \text { normal value }\end{array}$ \\
\hline $\begin{array}{l}\text { Doripenem* } \\
\text { Prod Info Doribax, } \\
2008(27)\end{array}$ & 3 & 0.05 & $\begin{array}{l}\text { Dehydropeptidase I, leading to an } \\
\text { open ring inactive metabolite }\end{array}$ & 0.3 & 8.1 & No adjustment required \\
\hline
\end{tabular}


Table II (Cont.). Recommendations for drug dosage in patients with chronic liver disease

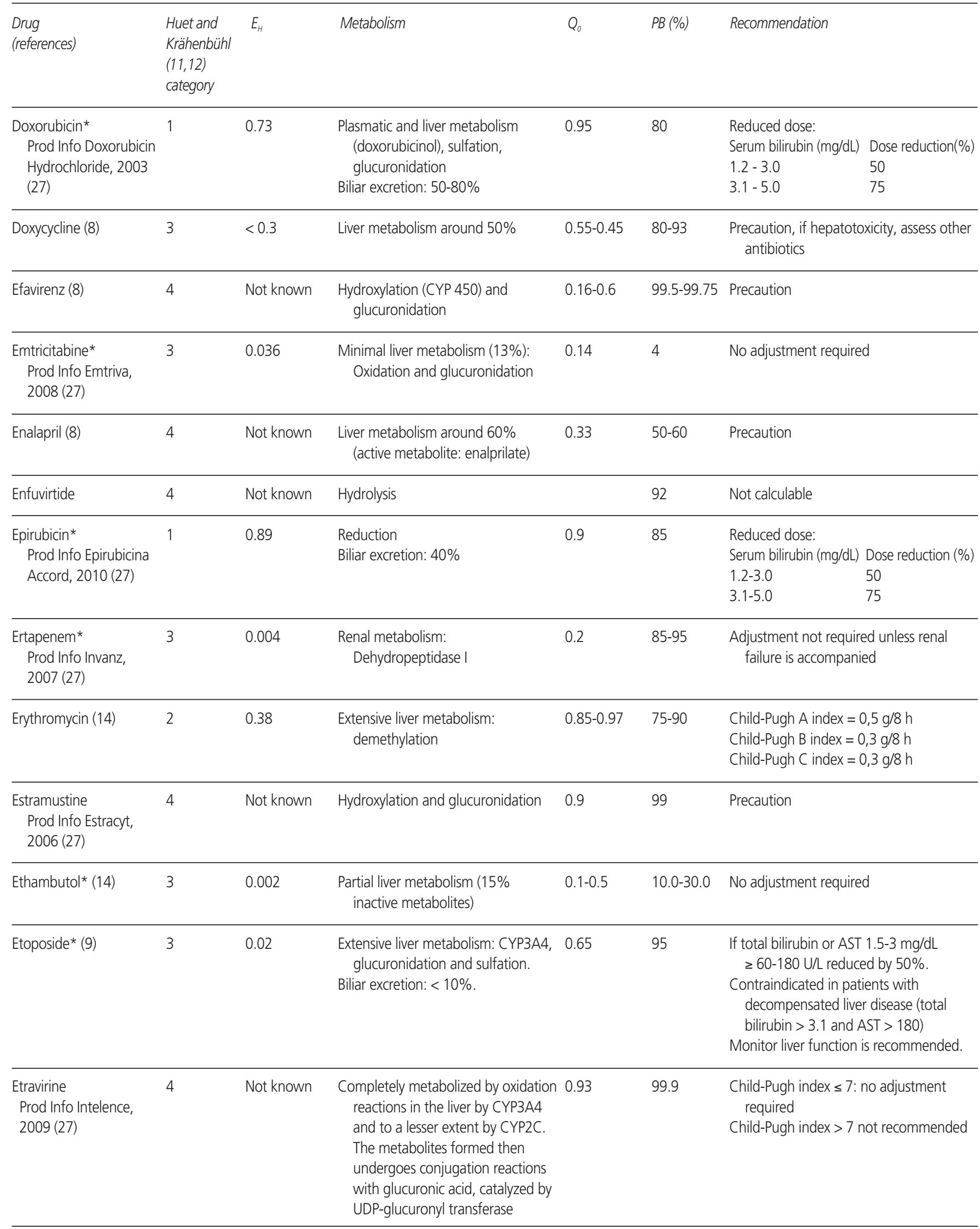


Table II (Cont.). Recommendations for drug dosage in patients with chronic liver disease

\begin{tabular}{|c|c|c|c|c|c|c|}
\hline $\begin{array}{l}\text { Drug } \\
\text { (references) }\end{array}$ & $\begin{array}{l}\text { Huet and } \\
\text { Krähenbühl } \\
(11,12) \\
\text { category }\end{array}$ & $E_{H}$ & Metabolism & $Q_{0}$ & $P B(\%)$ & Recommendation \\
\hline $\begin{array}{l}\text { Exemestane* } \\
\text { Prod Info Aromasin, } \\
2007 \text { (27) }\end{array}$ & 1 & 6.77 & $\begin{array}{l}\text { Oxidation (CYP3A) } \\
\text { Aldocetoreductase followed by } \\
\text { conjugation.Biliar excretion: } 40 \%\end{array}$ & 1 & 90 & Monitor liver functi \\
\hline
\end{tabular}

\begin{tabular}{|c|c|c|c|c|c|c|}
\hline Flucytosine (9) & 4 & Not known & - & $0.1-0.35$ & 4 & No adjustment required \\
\hline Fluconazole* (14) & 3 & 0.002 & Liver metabolism around 10\% & 0.2 & $11.0-12$ & Avoid in decompensated hepatitis \\
\hline Fludarabine* (9) & 3 & 0.06 & - & 0.35 & $10.0-30.0$ & No adjustment required \\
\hline Fluorouracil* (9) & 1 & 0.71 & Dihydropyrimidine dehydrogenase & 0.95 & 94 & $\begin{array}{l}\text { If total bilirubin } \leq 5 \mathrm{mg} / \mathrm{dL} \text { : } 100 \% \text { dose } \\
\text { If total bilirubin }>5 \mathrm{mg} / \mathrm{dL} \text { : avoid } \\
\text { In cirrhotic patients recommended starting } \\
\text { dose of } 50 \% \text { and increase as liver toxicity }\end{array}$ \\
\hline Fluoxetine* $(9)$ & 3 & 0.22 & $\begin{array}{l}\text { N- Demethylation (CYP2D6): active } \\
\text { metabolite (norfluoxetine) }\end{array}$ & 0.97 & 94 & $\begin{array}{l}\text { Reduce dose } 50 \% \text { in cirrhotics (without } \\
\text { ascites) or use alternate days }\end{array}$ \\
\hline $\begin{array}{l}\text { Fluphenazine* } \\
\text { Prod Info Modecate, } \\
2009(27)\end{array}$ & 2 & 0.47 & $\begin{array}{l}\text { Hydroxylation } \\
\text { Glucuronidation, sulfation } \\
\text { Demethylation biliar excretion and } \\
\text { enterohepatic circulation }\end{array}$ & 1 & 90 & Avoid Child-Pugh C index \\
\hline
\end{tabular}

\begin{tabular}{|c|c|c|c|c|c|c|}
\hline Flutamide (9) & 4 & Not known & Hydroxylation & 1 & 95 & No adjustment required \\
\hline $\begin{array}{l}\text { Fosamprenavir* } \\
\text { Prod Info Telzir, } 2004 \\
\text { (27) }\end{array}$ & 2 & 0.57 & Extensive liver metabolism: CYP3A4 & 0.86 & 90 & $\begin{array}{l}\text { Child-Pugh index 5-6: } 700 \mathrm{mg} / 12 \mathrm{~h}+ \\
\text { Ritonavir } 100 \mathrm{mg} / 24 \mathrm{~h} \\
\text { Child-Pugh index 7-9: } 450 \mathrm{mg} / 12 \mathrm{~h}+ \\
\text { Ritonavir } 100 \mathrm{mg} / 24 \mathrm{~h} \\
\text { Child-Pugh index 10-15: } 300 \mathrm{mg} / 12 \mathrm{~h}+ \\
\text { Ritonavir } 100 \mathrm{mg} / 24 \mathrm{~h}\end{array}$ \\
\hline
\end{tabular}

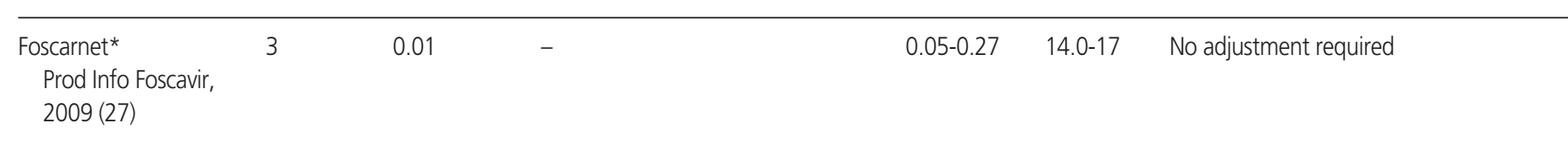

\begin{tabular}{|c|c|c|c|c|c|c|}
\hline Furosemide (8) & 4 & Not known & Liver metabolism around 10\% & $0.1-0.4$ & $91-99$ & Monitor electrolytes \\
\hline Ganciclovir* (9) & 3 & 0.002 & - & 0.86 & $1.0-2.0$ & No adjustment required \\
\hline $\begin{array}{l}\text { Gemcitabine* }^{*} \\
\text { Prod Info Gemcitabina } \\
\text { Stada, } 2010(27)\end{array}$ & 1 & 0.9 & $\begin{array}{l}\text { Deamination } \\
\text { Phosphorylation }\end{array}$ & 0.9 & $1.0-12$ & Precaution \\
\hline $\begin{array}{l}\text { Glibenclamide } \\
\text { Prod Info Micronase, } \\
1997 \text { (27) }\end{array}$ & 4 & Not known & Extensive liver metabolism & 0.5 & 99 & Start with $1.25 \mathrm{mg}$ and monitor effect \\
\hline $\begin{array}{l}\text { Goserelin* } \\
\text { Prod Info Zoladex, } \\
2010(27)\end{array}$ & 3 & 0.04 & - & 0.4 & 25 & No adjustment required \\
\hline
\end{tabular}


Table II (Cont.). Recommendations for drug dosage in patients with chronic liver disease

\begin{tabular}{|c|c|c|c|c|c|c|}
\hline $\begin{array}{l}\text { Drug } \\
\text { (references) }\end{array}$ & $\begin{array}{l}\text { Huet and } \\
\text { Krähenbühl } \\
(11,12) \\
\text { category }\end{array}$ & $E_{H}$ & Metabolism & $Q_{0}$ & $P B(\%)$ & Recommendation \\
\hline Griseofulvin (8) & 4 & Not known & Extensive liver metabolism & 0.8 & & Avoid Child-Pugh C index \\
\hline Haloperidol* (8) & 2 & 0.55 & $\begin{array}{l}\text { N-dealkylation: CYP3A4, CYP2D6 } \\
\text { Reduction: CYP 3A4, CYP } \\
\text { 2D6 } \\
\text { Glucuronidation. } \\
\text { Biliar excretion: 15\% } \\
\text { Enterohepatic circulation }\end{array}$ & 1 & 92 & $\begin{array}{l}\text { Reduce dose } 50 \% \text { (precipitated coma) } \\
\text { and monitor effect }\end{array}$ \\
\hline
\end{tabular}

Heparin
Prod Info Heparina
Hospira, 2007 (27)

Monitor dose based on activated partial thromboplastin time APTT

\begin{tabular}{lllll}
\hline Hydralazine (8) $\quad 4 \quad$ Not known $\quad$ Liver acetylation & 0.2 & $88-90 \quad \begin{array}{r}\text { Precaution, dose reduction is } \\
\text { recommended based on their toxicity }\end{array}$
\end{tabular}

\begin{tabular}{lllllll}
\hline Hydrochlorothiazide (9) & 4 & Not known & Not undergo hepatic metabolism & $0.3-0.5$ & 40 & Monitor electrolytes \\
\hline Ibuprofen* (8) & 3 & 0.001 & Extensive liver metabolism & 0.2 & 99 & Precaution \\
\hline Idarubicin (9) & 1 & 1 & $\begin{array}{c}\text { Extensive liver metabolism } \\
\text { idarubicinol }\end{array}$ & 0.4 & 96 & $\begin{array}{c}\text { If total bilirubin }=2.6-5 \text { dose reduction } \\
50 \% \text { Dot administer if total bilirubin }>5 \mathrm{mg} / \mathrm{dL}\end{array}$ \\
\end{tabular}

\begin{tabular}{|c|c|c|c|c|c|c|}
\hline Ifosfamide* (9) & 3 & 0.02 & Liver metabolism: CYP3A & 0.5 & & Monitor liver function \\
\hline $\begin{array}{l}\text { Imatinib } \\
\text { Prod Info Gleevec, } \\
2008(27)\end{array}$ & 4 & Not known & $\begin{array}{l}\text { N-demethylation: CYP 3A } \\
\text { Biliar excretion: } 20 \%\end{array}$ & 0.95 & 95 & $\begin{array}{l}\text { In patients Child-Pugh C index reduce } \\
\text { doses to } 25 \% \text {. Do not administer if total } \\
\text { bilirubin }>3 \text { normal or } \\
\text { transaminases }>5 \text { normal value }\end{array}$ \\
\hline
\end{tabular}

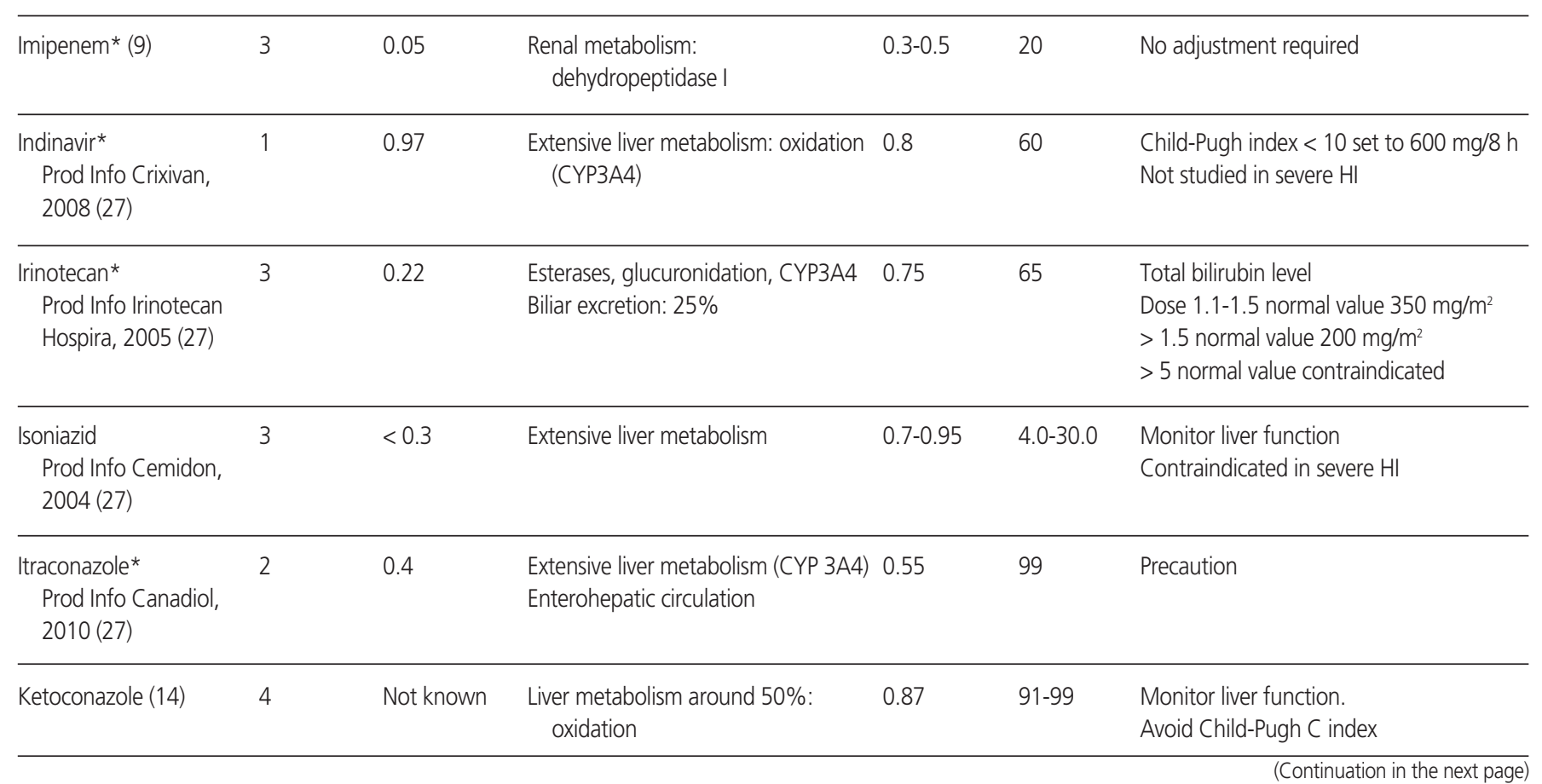


Table II (Cont.). Recommendations for drug dosage in patients with chronic liver disease

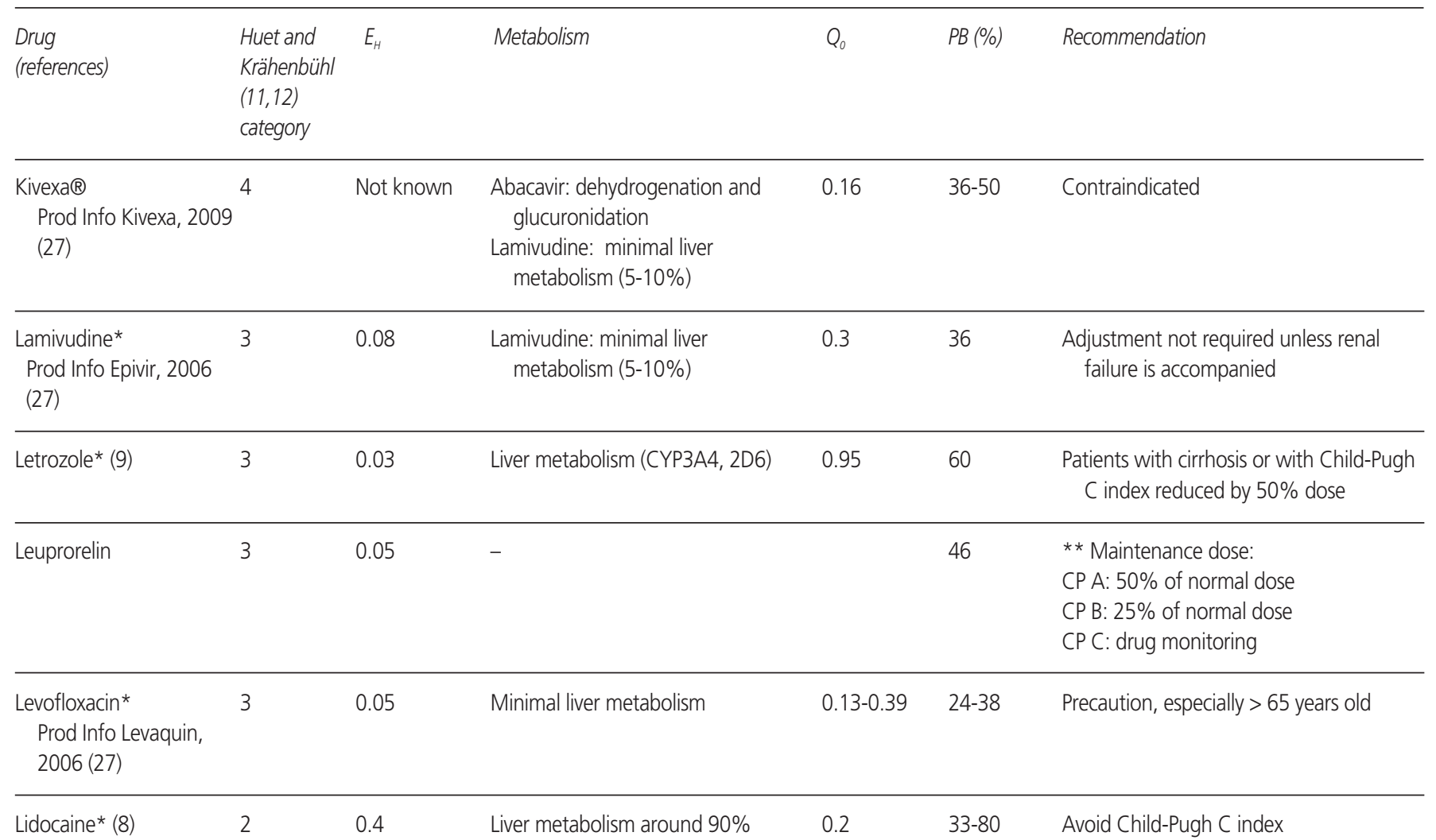

\begin{tabular}{lllllll}
\hline Linezolid* $^{*}$ & 3 & 0.06 & Minimal liver metabolism & 0.7 & 31 & No adjustment required \\
Prod Info Zyvoxid, & & & & & &
\end{tabular}

2010 (27)

\begin{tabular}{lllll}
\hline $\begin{array}{l}\text { Lopinavir/Ritonavir* } \\
\text { Prod Info Kaletra, } \\
2006(27)\end{array}$ & $0.01 \quad \begin{array}{c}\text { Lopinavir: extensive liver metabolism } \\
\text { (CYP 3A) } \\
\text { Ritonavir: extensive liver metabolism } \\
\text { (CYP3A4) }\end{array}$
\end{tabular}

\begin{tabular}{|c|c|c|c|c|c|c|}
\hline Maraviroc* & 2 & 0.4 & $\begin{array}{l}\text { Liver metabolism around } 58 \% \\
\text { (CYP3A4) }\end{array}$ & 0.76 & 76 & $\begin{array}{l}{ }^{*} \text { Initial dose: start at the minimum of } \\
\text { the normal dose } \\
\text { Maintenance dose: same that low } E_{H} \\
\text { and PB }\end{array}$ \\
\hline
\end{tabular}

\begin{tabular}{|c|c|c|c|c|c|c|}
\hline Megestrol & 4 & Not known & Glucuronidation & 1 & 18 & Not calculable \\
\hline Meglumine Antimonate (8) & 4 & Not known & - & 0.1 & & Precaution \\
\hline Melphalan* (9) & 2 & 0.31 & Hydroxylation & 0.9 & 80 & No adjustment required \\
\hline $\begin{array}{l}\text { Meropenem* } \\
\text { Prod Info Meronem, } \\
2010(27)\end{array}$ & 3 & 0.05 & - & 0.3 & 2 & No adjustment required \\
\hline
\end{tabular}


Table II (Cont.). Recommendations for drug dosage in patients with chronic liver disease

\begin{tabular}{|c|c|c|c|c|c|c|}
\hline $\begin{array}{l}\text { Drug } \\
\text { (references) }\end{array}$ & $\begin{array}{l}\text { Huet and } \\
\text { Krähenbühl } \\
(11,12) \\
\text { category }\end{array}$ & $E_{H}$ & Metabolism & $Q_{0}$ & $P B(\%)$ & Recommendation \\
\hline Methadone (8) & 3 & $<0.3$ & $\begin{array}{l}\text { Liver metabolism: P450 CYP3A4, } \\
\text { CYP2B6, CYP2C19, CYP2C9, } \\
\text { CYP2D6; } \\
\text { N-demethylation }\end{array}$ & & $71-88$ & Avoid Child-Pugh C index \\
\hline $\begin{array}{l}\text { Metformin } \\
\text { Prod Info Dianben, } \\
2010(27)\end{array}$ & 4 & Not known & Not undergo hepatic metabolism & 0.1 & & Avoid Child-Pugh C index \\
\hline Methotrexate* (9) & 3 & 0.005 & Liver metabolism around 10\% & 0.05 & 50 & $\begin{array}{l}\text { Total bilirubin AST level reduce level } \\
<3 \mathrm{mg} / \mathrm{dL} \text { and AST < } 180 \mathrm{Ul}: 0 \% \\
3.1-5 \mathrm{mg} / \mathrm{dL} \text { or AST> } 180 \mathrm{Ul}: 25 \% \\
>5 \mathrm{mg} / \mathrm{dL} \text { : contraindicated }\end{array}$ \\
\hline Methyldopa (8) & 4 & Not known & Liver metabolism around 50\% & $0.3-0.5$ & & Precaution \\
\hline Metoclopramide* (9) & 3 & 0.04 & $\begin{array}{l}\text { Minimal liver metabolism: sulfation } \\
\text { and glucuronidation }\end{array}$ & 0.1 & 30 & No adjustment required \\
\hline Metronidazole* (14) & 3 & 0.002 & $\begin{array}{l}\text { Liver metabolism around 50\%: } \\
\text { oxidation }\end{array}$ & $0.2-0.4$ & $<20$ & $\begin{array}{l}\text { Child-Pugh } A \text { and B index: unchanged } \\
\text { Child-Pugh C index: } 250 \mathrm{mg} / 8 \mathrm{~h}\end{array}$ \\
\hline Micafungin * (14) & 2 & 0.36 & Liver, extent unknown & 0.71 & 99 & $\begin{array}{l}\text { Monitor liver function. } \\
\text { Avoid Child-Pugh C index }\end{array}$ \\
\hline
\end{tabular}

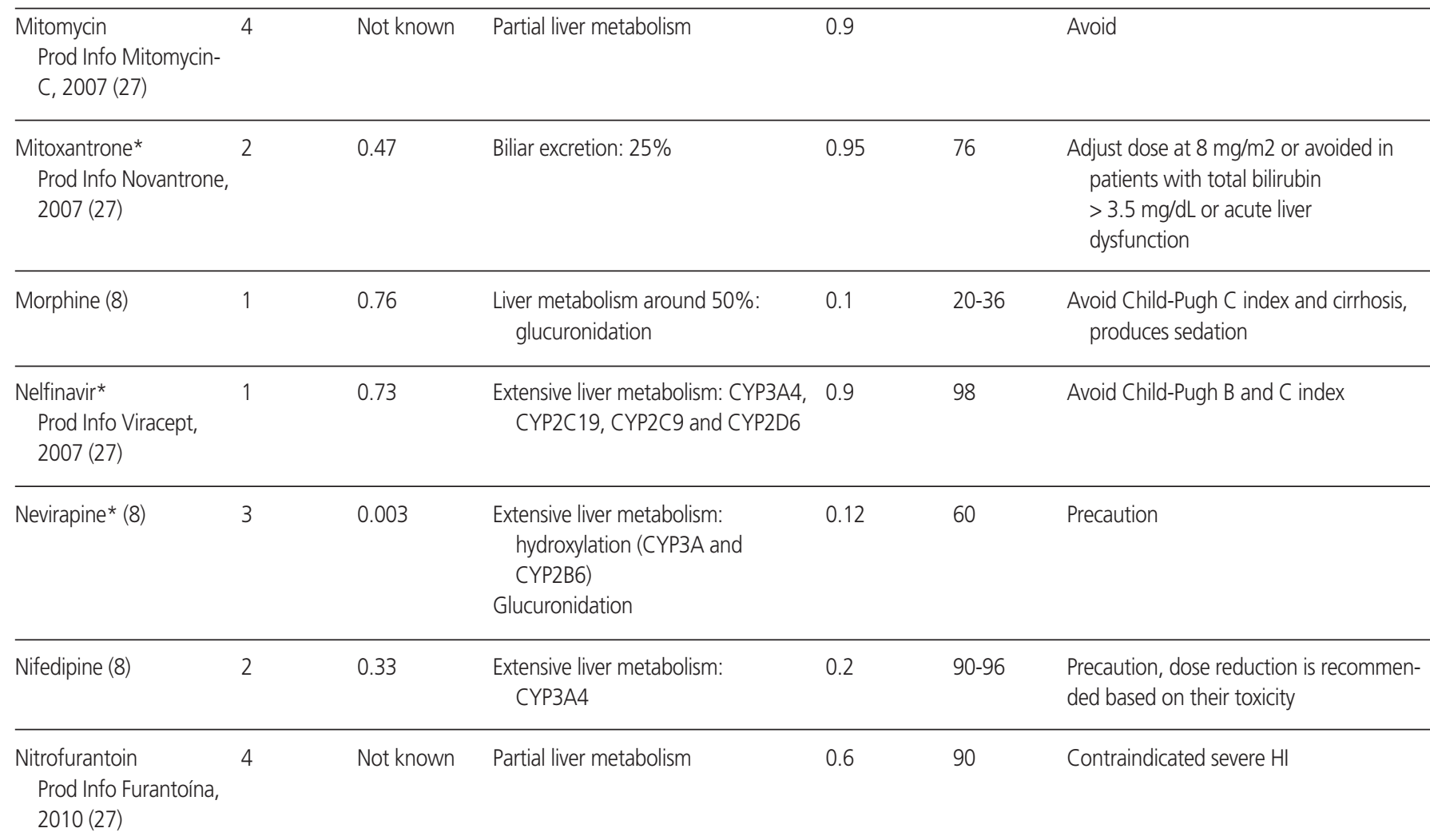


Table II (Cont.). Recommendations for drug dosage in patients with chronic liver disease

\begin{tabular}{|c|c|c|c|c|c|c|c|c|}
\hline $\begin{array}{l}\text { Drug } \\
\text { (references) }\end{array}$ & $\begin{array}{l}\text { Huet and } \\
\text { Krähenbühl } \\
(11,12) \\
\text { category }\end{array}$ & $E_{H}$ & Metabolism & $Q_{0}$ & $P B(\%)$ & \multicolumn{3}{|c|}{ Recommendation } \\
\hline $\begin{array}{l}\text { Ofloxacin } \\
\text { Prod Info Floxin, } 2006 \\
(27)\end{array}$ & 4 & Not known & Minimal liver metabolism & 0.1 & $20-32$ & \multicolumn{3}{|c|}{ Max. 400 mg/day } \\
\hline $\begin{array}{l}\text { Oxaliplatin } \\
\text { Prod Info Eloxatin, } \\
2007 \text { (27) }\end{array}$ & 4 & Not known & $\begin{array}{l}\text { Nonenzymatic reduction } \\
\text { Biliar excretion: } 5 \%\end{array}$ & 0.5 & 75 & \multicolumn{3}{|c|}{ No adjustment required } \\
\hline $\begin{array}{l}\text { Paclitaxel* }^{*} \\
\text { Prod Info Taxol, } 2010 \\
\text { (27) }\end{array}$ & 3 & 0.24 & $\begin{array}{l}\text { Extensive liver metabolism: CYP2C8, } \\
\text { and lesser extent by CYP3A4 } \\
\text { Biliar excretion: }>5 \%\end{array}$ & 0.95 & 95 & $\begin{array}{l}\text { Transaminase } \\
<2 \mathrm{Nv} \\
2-10 \mathrm{Nv} \\
<10 \mathrm{Nv} \\
>10 \mathrm{Nv} \\
<10 \mathrm{Nv} \\
<10 \mathrm{Nv} \\
<10 \mathrm{Nv} \\
>10 \mathrm{Nv} \\
\mathrm{Nv} \text { : normal val }\end{array}$ & $\begin{array}{l}\text { Total } \\
\text { bilirubin 2 } \\
<1.5 \mathrm{mg} / \mathrm{dL} \\
<1.5 \mathrm{mg} / \mathrm{dL} \\
1.6-7.5 \mathrm{mg} / \mathrm{dL} \\
>7.5 \mathrm{mg} / \mathrm{dL} \\
<1.25 \mathrm{Nv} \\
1.26-2 \mathrm{Nv} \\
2.01-5 \mathrm{Nv} \\
>5 \mathrm{Nv} \\
\mathrm{e}\end{array}$ & $\begin{array}{c}\text { Dose level } \\
24 \mathrm{~h} \text { infusion } \\
135 \mathrm{mg} / \mathrm{m}^{2} \\
100 \mathrm{mg} / \mathrm{m}^{2} \\
50 \mathrm{mg} / \mathrm{m}^{2} \\
\text { not administer } \\
3 \mathrm{~h} \text { nfusion } \\
175 \mathrm{mg} / \mathrm{m}^{2} \\
135 \mathrm{mg} / \mathrm{m}^{2} \\
90 \mathrm{mg} / \mathrm{m}^{2} \\
\text { not administer }\end{array}$ \\
\hline
\end{tabular}

\begin{tabular}{|c|c|c|c|c|c|c|}
\hline $\begin{array}{l}\text { Piperacillin Sodium/ } \\
\text { Tazobactam* }\end{array}$ & 3 & 0.02 & Partial liver metabolism & $0.2-0.3$ & 30 & $\begin{array}{l}\text { No dose adjustment is necessary, use } \\
\text { with precaution }\end{array}$ \\
\hline
\end{tabular}

Prod Info Tazocel,

$2010(27)$

\begin{tabular}{|c|c|c|c|c|c|c|}
\hline Phenytoin (9) & 3 & $<0.3$ & $\begin{array}{l}\text { Hydroxylation: CYP2C9, CYP2C19 } \\
\text { Glucuronidation }\end{array}$ & 1 & 90 & $\begin{array}{l}\text { Determine drug level } \\
\text { Increased toxicity risk }\end{array}$ \\
\hline
\end{tabular}

Glucuronidation

Phenobarbital, 2005

(27)

\begin{tabular}{|c|c|c|c|c|c|c|}
\hline $\begin{array}{l}\text { Posaconazole* } \\
\text { Prod Info Noxafil, } \\
2005 \text { (27) }\end{array}$ & 2 & 0.31 & Liver metabolism: glucuronidation & $0.85-0.86$ & 98 & Precaution \\
\hline Prednisolone (9) & 3 & $<0.3$ & Extensive liver metabolism & & $70-90$ & No adjustment required \\
\hline Procainamide (8) & 4 & Not known & Liver metabolism around 50\% & 0.1 & $10.0-20.0$ & Reduce by $25 \%$ or avoid in severe cirrhosis \\
\hline Promethazine * (8) & 1 & 0.76 & $\begin{array}{l}\text { Extensive liver metabolism: } \\
\text { sulfoxides and glucuronyl } \\
\text { conjugates }\end{array}$ & 1 & 85 & Avoid Child-Pugh C index \\
\hline
\end{tabular}


Table II (Cont.). Recommendations for drug dosage in patients with chronic liver disease

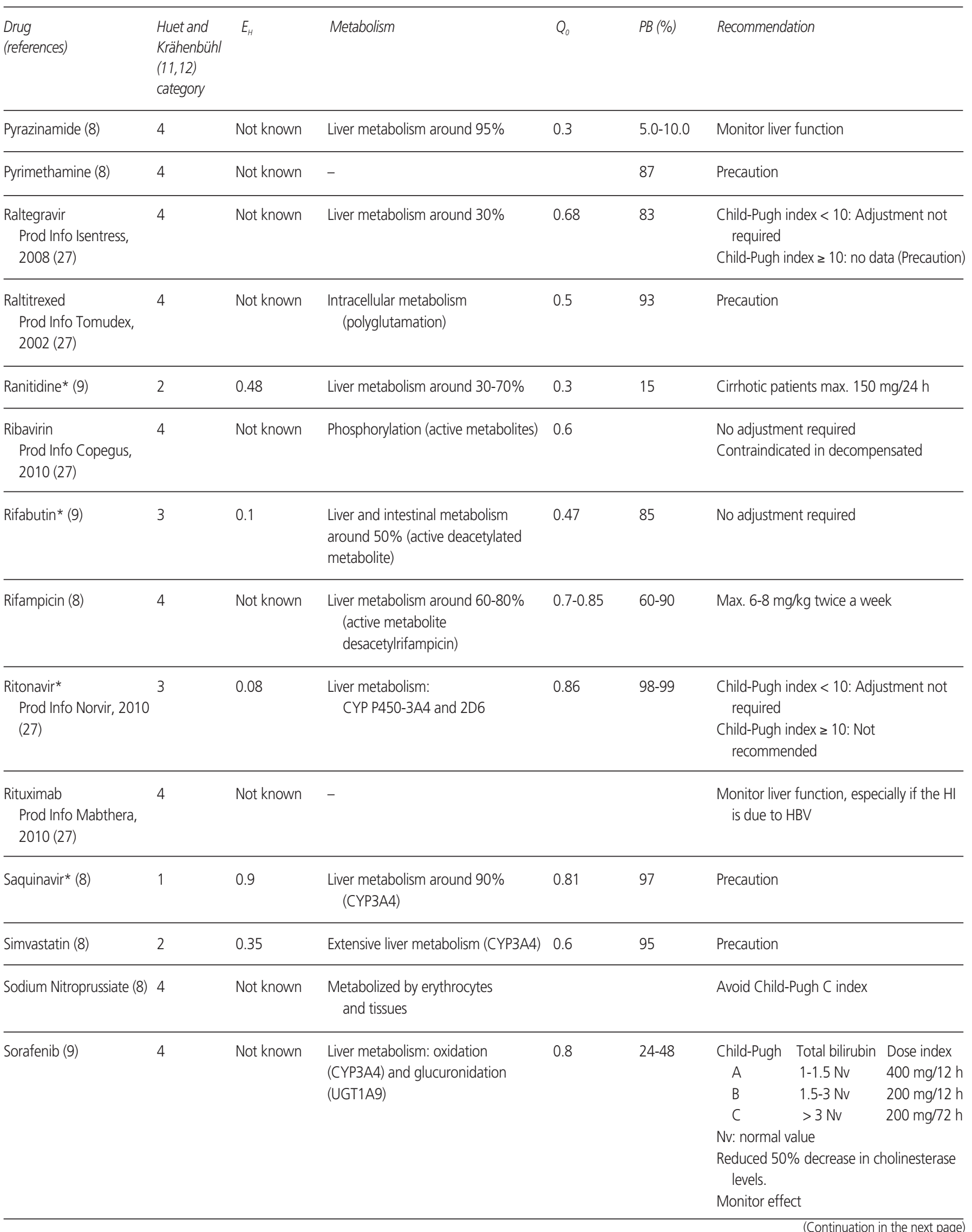


Table II (Cont.). Recommendations for drug dosage in patients with chronic liver disease

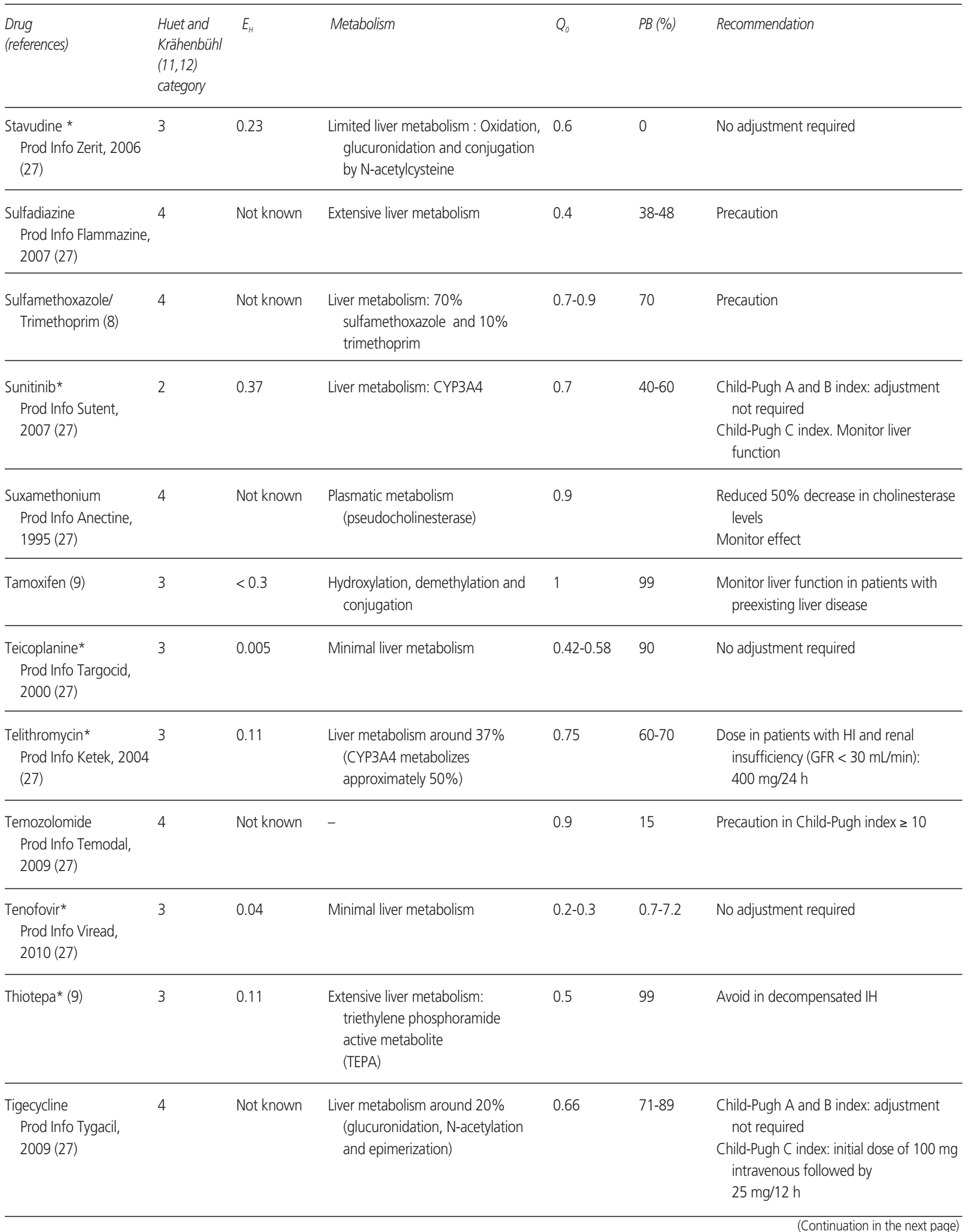


Table II (Cont.). Recommendations for drug dosage in patients with chronic liver disease

\begin{tabular}{|c|c|c|c|c|c|c|}
\hline $\begin{array}{l}\text { Drug } \\
\text { (references) }\end{array}$ & $\begin{array}{l}\text { Huet and } \\
\text { Krähenbühl } \\
(11,12) \\
\text { category }\end{array}$ & $E_{H}$ & Metabolism & $Q_{0}$ & $P B(\%)$ & Recommendation \\
\hline $\begin{array}{l}\text { Tioguanine } \\
\text { Prod Info Tioguanina } \\
\text { Glaxosmithkline, } \\
2007 \text { (27) }\end{array}$ & 4 & Not known & $\begin{array}{c}\text { Metabolism thiopurine } \\
\text { methyltransferase }\end{array}$ & $>0.9$ & $5.0-9.0$ & Monitor liver function \\
\hline $\begin{array}{l}\text { Tipranavir* } \\
\text { Prod Info Aptivus, } \\
2005 \text { (27) }\end{array}$ & 3 & 0.01 & Extensive liver metabolism: CYP3A4 & 0.82 & 99 & $\begin{array}{l}\text { Child-Pugh index }<6 \text { : adjustment not } \\
\text { required } \\
\text { Child-Pugh index } \geq 7 \text { : contraindicated }\end{array}$ \\
\hline Tobramycin * (9) & 3 & 0.0002 & $\begin{array}{l}\text { Not undergo hepatic } \\
\text { metabolism }\end{array}$ & $0.15-0.4$ & $0-30$ & No adjustment required \\
\hline $\begin{array}{l}\text { Topotecan* } \\
\text { Prod Info Hycamtin, } \\
2007 \text { (27) }\end{array}$ & 2 & 0.33 & $\begin{array}{l}\text { Hydrolysis } \\
\text { Biliar excretion: 20\% }\end{array}$ & 0.6 & 35 & No adjustment required \\
\hline Trastuzumab & 4 & Not known & - & 0.04 & & Not calculable \\
\hline $\begin{array}{l}\text { Trizivir® } \\
\text { Prod Info Trizivir, } \\
2009(27)\end{array}$ & 4 & Not known & $\begin{array}{l}\text { Abacavir: dehydrogenation and } \\
\text { glucuronidation } \\
\text { Lamivudine: minimal liver } \\
\text { metabolism (5-10\%) } \\
\text { Zidovudine: liver metabolism (60\%), } \\
\text { forming an inactive glucuronide } \\
\text { conjugate antiviral }\end{array}$ & 0.3 & $\begin{array}{l}50(\mathrm{~A}) \\
<36(\mathrm{~L}) \\
34-38(\mathrm{Z})\end{array}$ & Contraindicated \\
\hline $\begin{array}{l}\text { Truvada®* } \\
\text { Prod Info Truvada, } \\
2008(27)\end{array}$ & 3 & 0.08 & $\begin{array}{l}\text { Emtricitabine: minimal liver } \\
\text { metabolism }(13 \%)=\text { oxidation and } \\
\text { glucuronidation } \\
\text { Tenofovir: minimal liver } \\
\text { metabolism }\end{array}$ & $0.14-0.2$ & $\begin{array}{l}4(\mathrm{E}) ; \\
0.7(\mathrm{~T})\end{array}$ & No adjustment required \\
\hline $\begin{array}{l}\text { Valproic Acid* } \\
\text { Prod Info Depakene, } \\
2006(27)\end{array}$ & 3 & 0.01 & $\begin{array}{l}\text { Extensive liver metabolism: } \\
\text { glucuronoconjugation (50\%) } \\
\text { and } \beta \text {-oxidation (40\%) }\end{array}$ & 0.95 & 90 & $\begin{array}{l}\text { Reduce dose by } 50 \% \text {. } \\
\text { Determine drug level }\end{array}$ \\
\hline Vancomycin* (9) & 3 & 0.0002 & $\begin{array}{l}\text { Not undergo hepatic } \\
\text { metabolism }\end{array}$ & $0.0-0.6$ & $30-55$ & No adjustment required \\
\hline Verapamil (9) & 1 & 0.7 & Liver metabolism around 65-80\% & 0.3 & $88-94$ & Child-Pugh C index reduced by $50 \%$ \\
\hline
\end{tabular}

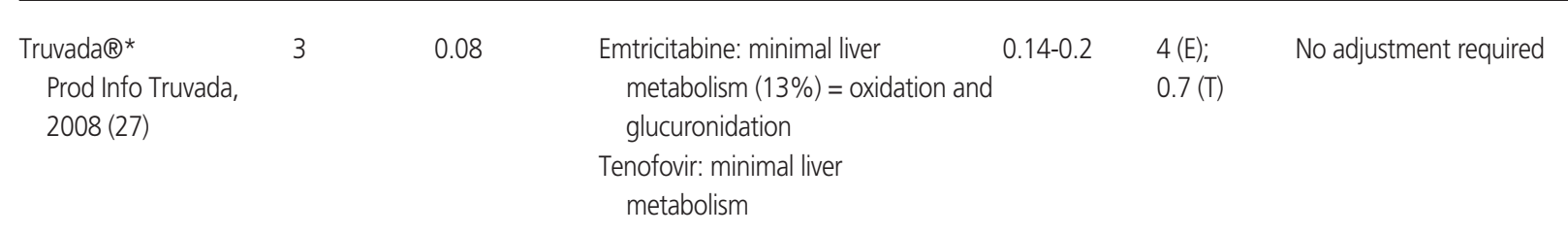

\begin{tabular}{|c|c|c|c|c|c|c|}
\hline $\begin{array}{l}\text { Valproic Acid* } \\
\text { Prod Info Depakene, } \\
2006 \text { (27) }\end{array}$ & 3 & 0.01 & $\begin{array}{l}\text { Extensive liver metabolism: } \\
\text { glucuronoconjugation (50\%) } \\
\text { and } \beta \text {-oxidation }(40 \%)\end{array}$ & 0.95 & 90 & $\begin{array}{l}\text { Reduce dose by } 50 \% \text {. } \\
\text { Determine drug level }\end{array}$ \\
\hline Vancomycin* (9) & 3 & 0.0002 & $\begin{array}{l}\text { Not undergo hepatic } \\
\text { metabolism }\end{array}$ & $0.0-0.6$ & $30-55$ & No adjustment required \\
\hline
\end{tabular}

1

Liver metabolism around 65-80\% $\quad 0.3$

$88-94$

Child-Pugh C index reduced by $50 \%$ of intravenous dose or $20 \%$ of oral doses

\begin{tabular}{|c|c|c|c|c|c|c|}
\hline Vinblastine (9) & 4 & Not known & $\begin{array}{l}\text { Liver metabolism: CYP3A4 } \\
\text { Biliar excretion: > 50\% }\end{array}$ & 1 & 75 & $\begin{array}{l}\text { Reduce dose by } 50 \% \text { if total bilirubin } \\
>3 \mathrm{mg} / \mathrm{dL}\end{array}$ \\
\hline $\begin{array}{l}\text { Vincristine* } \\
\text { Prod Info Oncovin, } \\
1999(27)\end{array}$ & 3 & 0.09 & $\begin{array}{l}\text { Extensive liver metabolism: CYP3A4 } \\
\text { Biliar excretion: } 70 \%\end{array}$ & 0.9 & 75 & $\begin{array}{l}\text { Reduce dose } 50 \% \text { if total bilirubin } \\
\quad>3 \mathrm{mg} / \mathrm{dL} \\
\text { Avoid administration if total bilirubin } \\
>3.1 \text { and AST > } 180 \mathrm{IU}\end{array}$ \\
\hline
\end{tabular}


Table II (Cont.). Recommendations for drug dosage in patients with chronic liver disease

\begin{tabular}{|c|c|c|c|c|c|c|}
\hline $\begin{array}{l}\text { Drug } \\
\text { (references) }\end{array}$ & $\begin{array}{l}\text { Huet and } \\
\text { Krähenbühl } \\
(11,12) \\
\text { category }\end{array}$ & $E_{H}$ & Metabolism & $Q_{0}$ & $P B(\%)$ & Recommendation \\
\hline Vindesine (9) & 4 & Not known & Extensive liver metabolism: CYP3A4 & & & $\begin{array}{l}50 \% \text { reduction in hepatobiliary } \\
\text { disease }\end{array}$ \\
\hline Warfarin (9) & 4 & Not known & $\begin{array}{l}\text { Extensive liver metabolism: } \\
\text { CYP2C9 , CYP2C19, CYP2C8, } \\
\text { CYP2C18, CYP1A2 and CYP3A4 }\end{array}$ & & 99 & Monitor prothrombin time (INR) \\
\hline
\end{tabular}

IU: international units. *HE calculated by Westphal et al. (11). **Delcò theoretical recommendation (1).

drugs with a low therapeutic margin and high-medium $\mathrm{E}_{\mathrm{H}}$ as measures to reduce the dose. Besides monitoring carefully the adverse effects and analyzing the possible pharmacological interactions. Finally, the article published by Azanza et al. (14) summarizes in tables the adjustments in the presence of renal insufficiency and/or of hepatic function failure from the antimicrobials according to the Child-Pugh score published in the literature. Drugs classified as "use with caution" are also another aspect to evaluate. The use of them should assess the balance of benefit/risk for each case because in all of them the adverse reactions in the hepatic level have been described, which could make the pathology worse in these subpopulation of patients. In the review, the lack of information and clinical studies with many drugs becomes clear. This is because until recent years, patients with Child-Pugh C were excluded in the development of new drugs for ethical reasons. Despite regulating agencies EMA and FDA recommend to study the research drugs in order to predict their behavior in patients with hepatic insufficiency and to being able to recommend dose adjustment, according to a recent survey, the number of drugs with specific 
adjustment recommendations based on the hepatic function with Child-Pugh scores is still very limited (24), probably due to hepatic insufficiency as exclusion criteria in most of the clinical trials. This would explain the lack of data in many cases in the SPC about dosage adjustment in moderate-severe hepatic insufficiency.

Moreover, there is a confirmed lack of consistency in the different consulted sources. This situation is also found in the general dosage recommendations as well as in the available kinetic data of drugs, mainly in the $\mathrm{E}_{\mathrm{H}}$, as it happens in the adjustment for renal insufficiency (25).

In view of the discrepancies highlighted above, the more conservative model is followed; despite it has the risk of an under-dosage, in some cases. Moreover, no guidelines in case of concomitant renal insufficiency or hepato-renal syndrome have been developed which could show one of its own limitations. Despite the existence of theoretical general recommendations in the medical literature about drug dosage in patients with hepatic insufficiency taking into account the pharmacokinetic parameters of $\mathrm{E}_{\mathrm{H}}$, plasma protein binding and bioavailability $(1,15-19,21,22)$, it has been considered more useful to individualize the recommendations for each drug and adjust them to adult patients with liver diseases. We wonder if the recommendations can be applied to all patients with hepatic disease or if, on the contrary, a limit between acute, chronic or multiorganic failure hepatic diseases should be established.

In clinical practice, patients should be carefully analyzed to determine the risks and benefits, taking many factors into account such as the severity of the disease, the consequences of not using the drug and the existence of equivalences or alternatives of different available treatments. When having the chance to choose among many drugs to treat the same disease, the less hepatotoxic should be selected through published reviews which would help to take the right one (26) and with a wide therapeutic range. In case of hepatic insufficiency, caution to manage the treatment is imposed as well as an effective followup to determine the intensity and duration of the desirable and undesirable effects, mainly if repeated or continuous administrations exist.

In conclusion, nowadays there are significant gaps in the necessary data for the safety in drug administration in patients with hepatic function failure. That is why in this review a contribution to the practical management of drugs to facilitate dosage recommendations to doctors/caregivers in patients with chronic liver disease is presented. It has been obtained through a synthesis of the published bibliography and completed by applying a theoretical methodology.

\section{REFERENCES}

1. Delcò F, Tchambaz L, Schlienger R, Drewe J, Krahenbuhl S. Dose adjustment in patients with liver disease. Drug Saf 2005;28: $529-45$.
2. Child CG, Turcotte JG. Surgery and portal hypertension. In: Child CG, editor. The Liver and Portal Hypertension. Philadelphia: Saunders; 1964. p. 50.

3. Pugh RN, Murray-Lyon IM, Dawson JL, Pietroni MC, Williams R. Transection of the oesophagus for bleeding oesophageal varices. Br J Surg 1973;60:646-9.

4. Wiesner R, Edwards E, Freeman R, Harper A, Kim R, Kamath P, et al. Model for end-stage liver disease (MELD) and allocation of donor livers. Gastroenterology 2003;124:91-6.

5. Cholongitas E, Marelli L, Shusang V, Senzolo M, Rolles K, Patch D, et al. A systematic review of the performance of the model for endstage liver disease (MELD) in the setting of liver transplantation. Liver Transpl 2006;12:1049-61.

6. Guidance for industry: pharmacokinetics in patients with impaired hepatic function: study design, data analysis, and impact on dosing and labelling. U.S. Department of Health and Human Services, Food and Drug Administration, Center for Drug Evaluation and Research and Center for Biologics Evaluation and Research. FDA, 2003. Disponible en http://www.fda.gov/cber/gdlns/imphep.pdf (fecha última consulta 15 de Abril de 2011).

7. Guideline on the evaluation of the pharmacokinetics of medicinal products in patients with impaired hepatic function. Committee for medicinal products for human use. EMA (CPMP/EWP/2339/02), 2005. Disponible en http://www.emea.europa.eu/pdfs/human/ewp/23390 (fecha última consulta 15 de Abril de 2011).

8. Stuart M, Kouimtzi M, Hill S, editors. WHO model formulary 2008. Geneva: WHO; 2008. Disponible en: http://www.who.int/selection_medicines/list/WMF2008.pdf (fecha última consulta 15 de Abril de 2011).

9. Drug Evaluation Monograph. En: Drugdex® Information System. Micromedex Inc. Engleewood: Staff; 2011. (fecha última consulta 15 de Abril de 2011).

10. Do Pazo F, Calvo C, Puigventós F, Ventayol P, Periañez L. Developing a search engine for pharmacoterapeutic information that is not published in biomedical journals. Farm Hosp 2011;35:254.e1-254.e5.

11. Huet PM, Villeneuve JP. Determinants of drug disposition in patients with cirrhosis. Hepatology 1983;3:913-8.

12. Krähenbühl S, Reichen J. Pharmacokinetics and pharmacodynamics in cirrhosis. Medicine 2002;30:24-7.

13. Westphal JF, Brogard JM. Drug administration in chronic liver disease. Drug Saf 1997;17:47-73.

14. Azanza JR, García E, Sádaba B, Manubens A. Uso de antimicrobianos en pacientes con insuficiencia renal o hepática. Enferm Infecc Microbiol Clin 2009;27:593-9.

15. Blaschke T.F. Effect of liver disease on dose optimization. International Congress Series 2001;1220:247-58.

16. Schlatter C, Egger SS, Tchambaz L, Krahenbuhl S. Pharmacokinetic changes of psychotropic drugs in patients with liver disease: implications for dose adaptation. Drug Saf 2009;32:561-78.

17. Pirmohamed M. Prescribing in liver disease. Medicine 2007;35:31-4.

18. Sloss A, Kluber P. Prescribing in liver disease. Aust Prescrib 2009;32:32-5.

19. Verbeeck RK. Pharmacokinetics and dosage adjustment in patients with hepatic dysfunction. Eur J Clin Pharmacol 2008;64:1147-61.

20. Shapiro J. "Hepatic dysfunction and drug dosing: The ABCs of the Child-Pugh Score". Drug \& Therapy bulletin 2005;19:1-3.

21. Kim JW, Hattori A, Phongsamran PV. Drug-induced liver disease. Crit Care Nurs Clin North Am 2010;22:323-34.

22. Drug use in liver impairment. Drug information service. Canterbury District Health Board. October 2003. http://www.druginformation.co.nz/Bulletins/DrugsLiverDisease.pdf (fecha última consulta 15 de Abril de 2011).

23. Klotz U. Antiarrhythmics: elimination and dosage considerations in hepatic impairment. Clin Pharmacokinet 2007;46:985-96.

24. Spray JW, Willett K, Chase D, Sindelar R, Connelly S. Dosage adjustment for hepatic dysfunction based on Child-Pugh scores. Am J Health Syst Pharm 2007;64:690,692-3.

25. Vidal L, Shavit M, Fraser A, Paul M, Leibovici L. Systematic comparison of four sources of drug information regarding adjustment of dose for renal function. BMJ 2005;331:263.

26. Lozano-Lanagrán M, Robles M, Lucena MI, Andrade RJ. Hepatotoxicity in 2011 advancing resolutely. Rev Esp Enferm Dig 2011;103:472-9. 
27. Centro de Información on-line de Medicamentos de la Agencia Española de Medicamentos y Productos Sanitarios (AEMPS), Ministerio de Sanidad, política social e igualdad. Acceso a fichas técnicas del medicamento aprobadas por la agencia española y por la Agencia Europea de Medicamentos (EMA). Disponibles en http://www.agemed.es y http://www.ema.europa.eu (fecha última consulta 15 de Abril de 2011) 